\title{
Preface to SPeCial ISSUe OF the Journal Of Automation, Mobile Robotics AND INTELLIGENT SYSTEMS ON RECENT ADVANCES IN INFORMATION TECHNOLOGY II
}

DOI: 10.14313/JAMRIS_1-2019/8

This issue of the Journal of Automation, Mobile Robotics and Intelligent Systems is devoted to selected aspects of current studies in the area of Information Technology - as presented by young talented contributors working in this field of research. This special issue is already the second edition of this series. Among included papers, one can find contributions dealing with the digital audio filter designing problem, building service-oriented systems, neural networks, modelling and classification. The idea of creating this special issue was born as a result of broad and interesting discussions during the Fifth Doctoral Symposium on Recent Advances in Information Technology (DS-RAIT 2018), held in Poznań (Poland) on September 9-12, 2018 as a satellite event of the Federated Conference on Computer Science and Information Systems (FedCSIS 2018). The aim of this meeting was to provide a platform for the exchange of ideas between early-stage researchers in Computer Science (PhD students in particular). Furthermore, the Symposium was to provide all participants an opportunity to obtain feedback on their ideas and explorations from the vastly experienced members of the IT research community who had been invited to chair all DS-RAIT thematic sessions. Therefore, submission of research proposals with limited preliminary results was strongly encouraged.

Here, we would like to individually mention the contribution entitled "UAV downwash dynamic texture features for terrain classification on autonomous navigation" written by João Pedro Carvalho, José Manuel Fonseca, and André Damas Mora. This contribution has received the Best Paper Award at DS-RAIT 2018.

This issue contains the following DS-RAIT papers in their special, extended versions.

The first paper, entitled The Design of Digital Audio Filter System used in Tomatis Method Stimulation, and authored by Krzysztof Jóźwiak, Michał Bujacz, and Aleksandra Królak, is a multidisciplinary research in which the authors merge the Tomatis Method (which is a rehabilitation technique used in psychology) with digital Electronic Ear systems, using an STM32F4 family micro-controller and ADC/DAC integrated circuits. This contribution is reviewing the Tomatis Method, as well as the main functions of the Electronic Ear, and describes the designed system with comparative measurements with the analogue original. In the opinion of editors and reviewers, this work apart from its practical values, might have extraordinary influence on the development of contemporary medicine.

Nikita Gerasimov, in his work entitled New approach to typified microservice composition and discovery, addresses the problems related to building service-oriented systems. One can name, among the aforementioned problems, the following issues: lack of static typing, lack of inter-service data type checking, as well as high services connectivity. In this contribution, the Author proposes a strong and static polymorphic type system and a type check algorithm. The latter technique was tested in a real service system, which demonstrated its reliability.

The last paper entitled Terrain classification using static and dynamic texture features by UAV downwash effect, was written by the team consisting of João Pedro Carvalho, José Manuel Fonseca, and André Damas Mora. In this paper, the Authors deal with identifying terrain type, in particular, they address this issue in the fields of autonomous navigation, mapping, decision-making and choice of emergency landing sites. The range of work and variety of techniques used to solve this problem is impressive. The authors start by acquiring real drone originating data, and then they analyze received real images and subsequently make the classification. In the opinion of the members of the Program Committee of DS-RAIT, this work, because of its multi-aspect nature, great presentation and promising results, was awarded the Best Paper of the event.

We would like to thank all those who were participating in, and contributing to the Symposium program, as well as all the authors who have submitted their papers. We also wish to thank all our colleagues, the members of the Program Committee, both for their hard work during the review process and for their cordiality and outstanding local organization of the Conference.

\section{Editors:}

\section{Piotr A. Kowalski}

Systems Research Institute, Polish Academy of Sciences

and

Faculty of Physics and Applied Computer Science,

AGH University of Science and Technology

\author{
Szymon Łukasik \\ Systems Research Institute, Polish Academy of Sciences \\ and \\ Faculty of Physics and Applied Computer Science, \\ AGH University of Science and Technology
}


